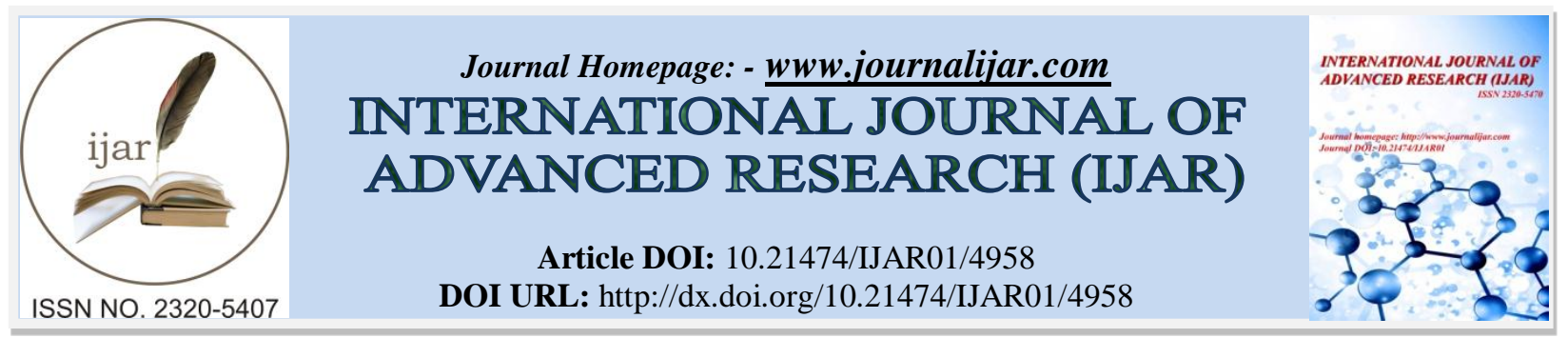

RESEARCH ARTICLE

\title{
DYNAMICAL BEHAVIORS OF FRACTIONAL ORDER PREY PREDATOR INTERACTIONS.
}

\author{
A. George maria selvam ${ }^{1}$, R. Dhineshbabu ${ }^{2}$ and S. Britto jacob ${ }^{1}$. \\ 1. Sacred Heart College, Tirupattur - 635601, S. India . \\ 2. DMI College of Engineering, Chennai - 600123, S. India.
}

\section{Manuscript Info}

Manuscript History

Received: 25 May 2017

Final Accepted: 27 June 2017

Published: July 2017

\section{Key words:-}

Fractional Order, LotkaVolterra Predator

Prey system, Discretization.

\section{Abstract}

This paper investigates the dynamical behavior of a Fractional order Prey - Predator interaction model. A discretization process is applied to obtain its discrete version. The fixed points are obtained and the stability properties are discussed.

\section{Introduction:-}

Recently, population models have received increasing attention by scientists due to their importance in ecology. Indeed, there are different approaches to study population models, e.g. ordinary differential equations, difference equations, partial differential equations and fractional order differential equations. Fractional - order differential equations (FOD) are used since they are naturally related to systems with memory which exists in most biological systems [2,4]. Many phenomena in population dynamics can be described successfully by the models using fractional order differential equations.

The fractional derivatives have several definitions. One of the most common definitions is the Caputo definition of fractional derivatives, which is often used in real applications. $D^{\alpha} f(t)=J^{l-\alpha} f^{l}(t), \alpha>0$, where $f^{l}$ represents the $l$-order derivative of $f(t), l=[\alpha]$ is the value of $\alpha$ rounded up to the nearest integer and $J^{q}$ is the $q$-order Riemann-Liouville integral operator $J^{q} h(t)=\int_{0}^{t} \frac{(t-\tau)^{(q-1)} h(\tau) d \tau}{\Gamma(q)}, q>0$, where $\Gamma($.$) is Euler's Gamma$ Function. The operator $D^{\alpha}$ is called the $\alpha$-order Caputo differential operator.

In this paper, we study the dynamical behaviors of fractional-order LotkaVolterra predator prey system. It is shown that the discretized fractional-order system produces a much richer set of patterns than those observed in the systems counterpart.

\section{Fractional Order Prey - Predator Model and its Discretization:-}

The Lotka Volterra equations, also known as the predator prey equations, are a pair of first-order, nonlinear, differential equations frequently used to describe the dynamics in which two species interact, one as a predator and the other as prey. The populations change through time according to the following pair of equations: 
$x^{\prime}=a x-b x y ; y^{\prime}=-c y+d x y$

where $x$ is the number of prey (for example rabbits), $y$ is the number of some predators (for example foxes) and $a, b, c, d$ are positive real parameters describing the interaction of two species: $a$-Growth rate of prey, $b$ - attack rate, $c$ - Predator mortality rate, $d$ - Growth rate of predator.

A more general model of predator prey interactions is the following system of differential equations,

$x^{\prime}=a x-b x y-e x^{2} ; y^{\prime}=-c y+d x y+f y^{2}$

Here the term $e x$ reflects the internal competition of the prey $x$ for their limited external resources and the term $f y$ reflects the competition among the predators for the limited number of prey. We shall consider the fractional order Lotka Volterra predator prey system which is given as follows

$D^{\alpha} x(t)=a x(t)-b x(t) y(t)-e x^{2}(t)$
$D^{\alpha} y(t)=-c y(t)+d x(t) y(t)+f y^{2}(t)$

where $t>0$ and $\alpha$ is the fractional order satisfying $\alpha \in(0,1]$.

Now, applying the discretization process for a fractional-order system described in $[3,8]$, we obtain the discrete fractional order predator prey system as follows:

$x_{n+1}=x_{n}+\frac{s^{\alpha}}{\alpha \Gamma(\alpha)}\left(x_{n}\left(a-b y_{n}-e x_{n}\right)\right) ; y_{n+1}=y_{n}+\frac{s^{\alpha}}{\alpha \Gamma(\alpha)}\left(y_{n}\left(-c+d x_{n}+f y_{n}\right)\right)$

Fixed Points:-

The fixed points of (1) are the points of intersections at which $D^{\alpha} x(t)=0$ and $D^{\alpha} y(t)=0$. We arrive at the following proposition. For the model system (1), there always exist trivial fixed point $E_{0}=(0,0)$, the semi-trivial fixed points $E_{1}=\left(\frac{a}{e}, 0\right), E_{2}=\left(0, \frac{c}{f}\right)$ and an interior point $E_{3}=\left(x^{*}, y^{*}\right)=\left(\frac{a f-b c}{e f-b d}, \frac{c e-a d}{e f-b d}\right)$.

Trivial and Semi-trivial fixed points and Stability Analysis;-

The Jacobian matrix of the linearized system of model (1) is

$$
J\left(x^{*}, y^{*}\right)=\left[\begin{array}{cc}
a-b y^{*}-2 e x^{*} & -b x^{*} \\
d y^{*} & -c+d x^{*}+2 f y^{*}
\end{array}\right] \text {. }
$$

Theorem 1. The trivial fixed point $E_{0}$ is locally asymptotically stable if $a<1, c<1$ otherwise unstable.

Proof: The Jacobian matrix at $E_{0}$ is given by

$$
J\left(E_{0}\right)=\left[\begin{array}{cc}
a & 0 \\
0 & -c
\end{array}\right]
$$

Hence the eigenvalues of $J\left(E_{0}\right)$ are $\lambda_{1}=a$ and $\lambda_{2}=-c$. Thus $E_{0}$ is stable when $a<1, c<1$. Otherwise $E_{0}$ is unstable trivial fixed point.

Theorem 2. The semi-trivial fixed point $E_{1}$ is locally asymptotically stable if $\frac{e(c-1)}{d}<a<\frac{e(c+1)}{d}$, otherwise unstable.

Proof: The Jacobian matrix at $E_{1}$ is given by 


$$
J\left(E_{1}\right)=\left[\begin{array}{cc}
-a & \frac{-a b}{e} \\
0 & \frac{a d-c e}{e}
\end{array}\right] .
$$

The eigen values of the matrix $J\left(E_{1}\right)$ are $\lambda_{1}=-a$ and $\lambda_{2}=\frac{a d-c e}{e}$. Hence $E_{1}$ is locally asymptotically stable when $\frac{e(c-1)}{d}<a<\frac{e(c+1)}{d}$ and unstable when $a>\frac{e(c+1)}{d}$ and $a<\frac{e(c-1)}{d}$.

Theorem 3. The semi-trivial fixed point $E_{2}$ is locally asymptotically stable if $\frac{f(a-1)}{b}<c<\frac{f(a+1)}{b}$, otherwise unstable.

Proof: The Jacobian matrix at $E_{2}$ is given by

$$
J\left(E_{2}\right)=\left[\begin{array}{cc}
\frac{a f-b c}{f} & 0 \\
\frac{d c}{f} & c
\end{array}\right] .
$$

The eigenvalues of the matrix $J\left(E_{2}\right)$ are $\lambda_{1}=\frac{a f-b c}{f}$ and $\lambda_{2}=c$. Hence $E_{2}$ is locally asymptotically stable when $\frac{f(a-1)}{b}<c<\frac{f(a+1)}{b}$ and unstable when $c>\frac{f(a+1)}{b}$ and $c<\frac{f(a-1)}{b}$.

\section{Interior fixed point and its Stability}

Here we investigate the linear stability of (1) at the interior fixed point $E_{3}$. Therefore, the corresponding Jacobian matrix at the interior fixed point $E_{3}$ can be easily expressed in the form

$$
J\left(E_{3}\right)=\left[\begin{array}{cc}
A & -B \\
C & D
\end{array}\right] \text {. }
$$

where $A=\frac{b c e-a e f}{e f-b d}, B=\frac{b(a f-b c)}{e f-b d}, C=\frac{d(c e-a d)}{e f-b d}$ and $D=\frac{c e f-a d f}{e f-b d}$.

The eigenvalues of the matrix $J\left(E_{3}\right)$ are $\lambda_{1,2}=\frac{(A+D) \pm \sqrt{(A-B)^{2}-4 B C}}{2}$. Hence $E_{3}$ is locally asymptotically stable when $\beta<1$ and unstable when $\beta>1$, where $\beta=\frac{(A+D) \pm \sqrt{(A-B)^{2}-4 B C}}{2}$.

\section{Dynamical behaviors of the Discretized Fractional Order Model:-}

Let us investigate the dynamics of the discretized fractional - order LotkaVolterra predator prey model (2). The dynamical behaviors of model (2) is determined by five parameters $a, b, c, d, e, f, s$ and $\alpha$.

Here we discuss the stability of fixed points of (2). The Jacobian matrix for (2) evaluated at any fixed point $\left(x^{*}, y^{*}\right)$ is given by 


$$
J\left(x^{*}, y^{*}\right)=\left[\begin{array}{cc}
1+\frac{s^{\alpha}}{\alpha \Gamma(\alpha)}\left(a-b y^{*}-2 e x^{*}\right) & -\frac{s^{\alpha}}{\alpha \Gamma(\alpha)} b x^{*} \\
\frac{s^{\alpha}}{\alpha \Gamma(\alpha)} d y^{*} & 1+\frac{s^{\alpha}}{\alpha \Gamma(\alpha)}\left(-c+d x^{*}+2 f y^{*}\right)
\end{array}\right]
$$

The characteristic equation of the Jacobian matrix can be written as

$\lambda^{2}-\operatorname{Tr} \lambda+\operatorname{Det}=0$

where $T r$ is the trace and Det is the determinant of the Jacobian matrix $J\left(x^{*}, y^{*}\right)$ and they are given as

$$
\begin{aligned}
& \operatorname{Tr}=2+\frac{s^{\alpha}}{\alpha \Gamma(\alpha)}\left(x^{*}(d-2 e)+y^{*}(2 f-b)+(a-c)\right) \\
& D e t=1+\frac{s^{\alpha}}{\alpha \Gamma(\alpha)}\left(x^{*}(d-2 e)+y^{*}(2 f-b)+(a-c)\right)+\left(\frac{s^{\alpha}}{\alpha \Gamma(\alpha)}\right)^{2}\left(x^{*}(a d+2 c e)+y^{*}(b c+2 a f)-2\left(d e x^{* 2}+b f y^{* 2}+2 e f x^{*} y^{*}\right)-a c\right)
\end{aligned}
$$

In order to study stability analysis of the fixed points of the model (2), we give the following theorems that can be easily proved by using the relation between roots and coefficients of the characteristic equation (3).

Theorem 1.If $0<s<\sqrt[\alpha]{\frac{2 \alpha \Gamma(\alpha)}{c}}$ then the fixed point $E_{0}$ is a saddle, If $s>\alpha \sqrt{\frac{2 \alpha \Gamma(\alpha)}{c}}$ then $E_{0}$ is a source and if $s=\sqrt[\alpha]{\frac{2 \alpha \Gamma(\alpha)}{c}}$, then $E_{0}$ is non-hyperbolic.

Proof: The Jacobian matrix $J$ at $E_{0}$ is given by

$$
J\left(E_{0}\right)=\left[\begin{array}{cc}
1+\frac{s^{\alpha}}{\alpha \Gamma(\alpha)} a & 0 \\
0 & 1-\frac{s^{\alpha}}{\alpha \Gamma(\alpha)} c
\end{array}\right] .
$$

Hence the eigenvalues are $\lambda_{1}=1+\frac{s^{\alpha}}{\alpha \Gamma(\alpha)} a$ and $\lambda_{2}=1-\frac{s^{\alpha}}{\alpha \Gamma(\alpha)} c$. Since $a>0$, then $\left|\lambda_{1}\right|>1$. Thus the fixed point $E_{0}$ is a saddle point if $0<s<\sqrt[\alpha]{\frac{2 \alpha \Gamma(\alpha)}{c}}$, source if $s>\alpha \sqrt{\frac{2 \alpha \Gamma(\alpha)}{c}}$ and non-hyperbolic if $s=\sqrt[\alpha]{\frac{2 \alpha \Gamma(\alpha)}{c}}$

Theorem 2. If $0<s<\sqrt[\alpha]{\frac{2 \alpha \Gamma(\alpha)}{a}}$, then the fixed point $E_{1}$ is a saddle point. If $s>\alpha \sqrt{\frac{2 \alpha \Gamma(\alpha)}{a}}$, then $E_{1}$ is source and if $s=\sqrt[\alpha]{\frac{2 \alpha \Gamma(\alpha)}{a}}$, then $E_{1}$ is non-hyperbolic.

Proof: The Jacobian matrix $J$ at $E_{1}$ is given by 


$$
J\left(E_{1}\right)=\left[\begin{array}{cc}
1-\frac{s^{\alpha}}{\alpha \Gamma(\alpha)} a & -\frac{s^{\alpha}}{\alpha \Gamma(\alpha)}\left(\frac{a b}{e}\right) \\
0 & 1+\frac{s^{\alpha}}{\alpha \Gamma(\alpha)}\left(\frac{a d-c e}{e}\right)
\end{array}\right]
$$

Hence, the eigenvalues are $\lambda_{1}=1-\frac{s^{\alpha}}{\alpha \Gamma(\alpha)} a$ and $\lambda_{2}=1+\frac{s^{\alpha}}{\alpha \Gamma(\alpha)}\left(\frac{a d-c e}{e}\right)$. Since $c, d>0$ then $\left|\lambda_{2}\right|>1$.

Thus the fixed point $E_{1}$ is a saddle point if $0<s<\sqrt[\alpha]{\frac{2 \alpha \Gamma(\alpha)}{a}}$, source if $s>\sqrt[\alpha]{\frac{2 \alpha \Gamma(\alpha)}{a}}$ and non-hyperbolic if $s=\sqrt[\alpha]{\frac{2 \alpha \Gamma(\alpha)}{a}}$

Theorem 3. If $\left|\lambda_{1}\right|>1$ and $\left|\lambda_{2}\right|>1$, then the fixed point $E_{2}$ is a source point. If $\left|\lambda_{1}\right|=1$ and $\left|\lambda_{2}\right|=1$, then $E_{2}$ is non-hyperbolic.

Proof: The Jacobian matrix $J$ at $E_{2}$ is given by

$$
J\left(E_{2}\right)=\left[\begin{array}{cc}
1+\frac{s^{\alpha}}{\alpha \Gamma(\alpha)} \frac{a f-b c}{f} & 0 \\
\frac{s^{\alpha}}{\alpha \Gamma(\alpha)} \frac{c d}{f} & 1+\frac{s^{\alpha}}{\alpha \Gamma(\alpha)} c
\end{array}\right] .
$$

Hence, the eigenvalues are $\lambda_{1}=1+\frac{s^{\alpha}}{\alpha \Gamma(\alpha)} \frac{a f-b c}{f}$ and $\lambda_{2}=1+\frac{s^{\alpha}}{\alpha \Gamma(\alpha)} c$. Since $a, c>0$, thus the fixed point $E_{2}$ is a source point if $\left|\lambda_{1}\right|>1$ and $\left|\lambda_{2}\right|>1$, non-hyperbolic if $\left|\lambda_{1}\right|=1$ and $\left|\lambda_{2}\right|=1$.

Theorem 4. The positive fixed point $E_{3}$ of the system (2) is locally asymptotically stable if $\gamma<0$, where $\gamma=\frac{s^{\alpha}}{\alpha \Gamma(\alpha)} \beta$ such that $\beta=\frac{(A+D) \pm \sqrt{(A-B)^{2}-4 B C}}{2}$.

Proof: The Jacobian matrix evaluated at the fixed point $E_{3}$ has the form

$$
J\left(E_{3}\right)=\left[\begin{array}{cc}
1+\frac{s^{\alpha}}{\alpha \Gamma(\alpha)} A & -\frac{s^{\alpha}}{\alpha \Gamma(\alpha)} B \\
\frac{s^{\alpha}}{\alpha \Gamma(\alpha)} C & 1+\frac{s^{\alpha}}{\alpha \Gamma(\alpha)} D
\end{array}\right] .
$$

The trace and determinant of the Jacobian matrix $J\left(E_{3}\right)$ is given by

$$
\begin{aligned}
& \operatorname{Tr}\left(J\left(E_{3}\right)\right)=2+\frac{s^{\alpha}}{\alpha \Gamma(\alpha)}(A+D) \\
& \operatorname{Det}=1+\frac{s^{\alpha}}{\alpha \Gamma(\alpha)}(A+D)+\left(\frac{s^{\alpha}}{\alpha \Gamma(\alpha)}\right)^{2}(A D+B C)
\end{aligned}
$$

Hence, the eigenvalues are $\lambda_{1,2}=1+\frac{s^{\alpha}}{\alpha \Gamma(\alpha)} \beta$. Thus $E_{3}$ is locally asymptotically stable when $\gamma<0$. 
The next result is obtained from theorem (4) immediately.

Lemma 1. The positive fixed point $E_{3}$ of the system (2) is unstable if $\gamma>0$, where $\gamma=\frac{s^{\alpha}}{\alpha \Gamma(\alpha)} \beta$ such that $\beta=\frac{(A+D) \pm \sqrt{(A-B)^{2}-4 B C}}{2}$ holds.

\section{References:-}

1. Abd-Elalim A. Elsadany, H. A. EL-Metwally, E. M. Elabbasy, H. N. Agiza, Chaos and bifurcation of a nonlinear discrete prey-predator system, Computational Ecology and Software, 2012, 2(3):169-180.

2. Berryman A.A, The origins and evolution of predator prey theory. Ecology 73, 15301535 (1992).

3. A. George Maria Selvam, R. Janagaraj, R. Dhineshbabu and Britto Jacob. S, Fractional Order Nonlinear Prey Predator Interactions, International Journal of Computational and Applied Mathematics. ISSN 1819-4966 Volume 12, Number 2 (2017), pp. 495-502 @ Research India Publications http://www.ripublication.com.

4. Ivo Petras, Fractional order Nonlinear Systems-Modeling, Analysis and Simulation, Higher Education Press, Springer International Edition, April 2010.

5. Leah Edelstein-Keshet, Mathematical Models in Biology, SIAM, Random House, New York, 2005.

6. El-Sayed AMA, El-Mesiry A, El-Saka H, On the fractional order logistic equation, Appl. Math. Lett. 20, 817823 (2007).

7. El-Sayed AMA, On the fractional differential equations, J. Appl. Math. Comput. 49(2-3), 205-213, (1992).

8. J. D. Murray, Mathematical Biology-I: An Introduction, 3-e, Springer International Edition, 2004.

9. Ravi P Agarwal, Ahmed MA El-Sayed and Sanaa M Salman, Fractional order Chua's system: discretization, bifurcation and chaos, Agarwal et al. Advances in Difference Equations, 2013, 2013:320. 